Research Article

\title{
Experimental Evidence of Precipitation of All 12 Variants in a Single $\beta$ Grain in Titanium Alloys
}

\author{
Yi Chen $\mathbb{D}^{1},{ }^{1,2,3,4}$ Hongchao Kou, ${ }^{5}$ Liang Cheng, ${ }^{1}$ Yunlong Zhang, ${ }^{1}$ Yun Yu, ${ }^{1}$ Yalin Lu, \\ and Fuxian $\mathrm{Zhu}^{1}$ \\ ${ }^{1}$ School of Materials and Engineering, Jiangsu University of Technology, Changzhou 213001, China \\ ${ }^{2}$ IMDEA Materials Institute, Madrid 28040, Spain \\ ${ }^{3}$ Polytechnic University of Madrid, E.T.S. de Ingenieros de Caminos 28040, Madrid, Spain \\ ${ }^{4}$ Sunnywell (China) New Material Technology Co., Ltd., Changzhou 213000, China \\ ${ }^{5}$ State Key Laboratory of Solidification Processing, Northwestern Polytechnical University, Xi'an 710072, China \\ Correspondence should be addressed to Yi Chen; webchenyi@hotmail.com
}

Received 28 May 2018; Revised 5 September 2018; Accepted 25 October 2018; Published 11 November 2018

Academic Editor: Michael J. Schütze

Copyright $\odot 2018$ Yi Chen et al. This is an open access article distributed under the Creative Commons Attribution License, which permits unrestricted use, distribution, and reproduction in any medium, provided the original work is properly cited.

\begin{abstract}
The effect of the changing of the local composition of the $\beta$ matrix on the precipitation of the $\alpha$ phase has been investigated by electron backscatter diffraction (EBSD) to obtain more insight in the nucleation and variant selection of these $\alpha$ plates based on the Ti-5.04Al/Ti-1.52Mo (at.\%) diffusion couple. The results showed that the composition gradient was formed from one side of the diffusion couple to another side after diffusion annealing. Followed by a secondary heat treatment process, it was found for the first time that all 12 variants formed in a single $\beta$ grain in the diffusion zone in the Ti-5.04Al/Ti-1.52Mo diffusion couple, which indicated that the changing of the local composition of the $\beta$ matrix significantly weakened the $\alpha$ variants selection behavior.
\end{abstract}

\section{Introduction}

The requirement of the high-performance airplane accelerates the application of titanium alloys due to their high specific strength together with the extraordinary corrosion resistance $[1,2]$. It is well known that the precipitate of the $\alpha$ phase from $\beta$ grains directly affects the properties of the titanium alloys, which results in a considerable amount of interest in the precipitation mechanism of the $\alpha$ phase [3-6].

During the $\beta$ to $\alpha$ phase transformation process, the $\alpha$ precipitates are well known to be formed according to the Burgers orientation relationship (BOR), which implies the parallelism of dense planes and direction of both phases: $(011)_{\beta} / /(0001)_{\alpha}$ and $[1-11]_{\beta} / /[11-20]_{\alpha}[7,8]$. Because of the symmetry of the $\alpha$ and $\beta$ phases, 12 crystallographic $\alpha$ orientations can form in a single parent $\beta$ grain, called $12 \alpha$ variants. When all $\alpha$ variants in a $\beta$ grain occur with equal statistical probability, the transformation is said to evolve without variant selection [3]. However, it is unable to observe all 12 variants in a $\beta$ grain in real alloys, which means the variant selection is always taking place during transformation in titanium alloys $[6,9,10]$. The variant selection will then result in the strong $\alpha$ transformation texture. Due to the high anisotropy, the mechanical properties of titanium alloys depend strongly on the texture of the $\alpha$ phase caused by the variant selection $[11,12]$. It is indicated that the random $\alpha$ texture distribution in the $\beta$ matrix will significantly improve the fatigue resistance and creep performance of titanium alloys $[13,14]$. Therefore, the weakening of variants election during thermomechanical processing plays a key role to control the $\alpha$ texture in the final products and enhance their mechanical properties.

A variety of factors could contribute to variant selection during $\alpha$ precipitation, including external or residual stresses on the $\beta$ matrix caused by the thermomechanical processing [15], heterogeneous nucleation on preexisting defects in the $\beta$ matrix, and so on $[5,16,17]$. However, recent researches show that introducing those factors will accelerate the variant selection [18-20]. For example, the effect of the stresses on the variant selection was proposed by Shi et al. [18]. In this work, 
it was found that the interplay between externally applied stress or strain and internal stress generated by the precipitation reaction itself will result in the variant selection due to the difference of the elastic interaction energy of each variant. Furthermore, Qui et al [20] found that the dislocations on the $\beta$ matrix significant influence the variant selection during $\alpha$ precipitation in titanium alloys due to the elastic interaction between $\alpha$ precipitates and dislocations.

Based on the phase transformation theory, the nucleation of $\alpha$ from $\beta$ matrix should first satisfy the composition fluctuation condition [21]. Therefore, the local composition of the $\beta$ matrix also will affect the nucleation of the $\alpha$ phase. Due to the existence of limited work in the literature, the main objective of this paper is to study the effect of the changing of local composition of the $\beta$ matrix on the precipitation of the $\alpha$ variant.

In this paper, the changing of local composition of the $\beta$ matrix was performed by the diffusion annealing of the Ti5.04Al/Ti-1.52Mo (at.\%) diffusion couple. Then, after a secondary heat treatment, the precipitation of $\alpha$ in the $\beta$ matrix was studied.

\section{Experimental}

In our previous study [22], a new high throughput method named "kinetic diffusion couple" (KDC) was designed for rapidly mapping microstructural and mechanical properties in Ti-Al-Mo systems by using the same Ti-5.04Al/Ti-1.52Mo (at.\%) diffusion couple studied in this paper. Thus, we followed the methods of our previous study [22] for preparing the diffusion couple in this paper, and the details were as follows. The Ti-5.04Al (at.\%) and Ti-1.52Mo (at.\%) alloys of the $\mathrm{Ti}-5.04 \mathrm{Al} / \mathrm{Ti}-1.52 \mathrm{Mo}$ (at.\%) diffusion couple were prepared from $99.9 \%$ sponge $\mathrm{Ti}, 99.99 \% \mathrm{Al}$, and $99.9 \% \mathrm{Mo}$ (mass \%) by arc melting in an argon atmosphere. The arc melting was repeated ten times to attain a homogeneous composition. The ingots were solid-solutioned at $1200^{\circ} \mathrm{C}$ for 8 hours under vacuum followed by water quenching for the further homogenization. The small cylinder samples of the $\mathrm{Ti}-5.04 \mathrm{Al}$ and $\mathrm{Ti}-1.52 \mathrm{Mo}$ alloys were cut from the ingots by a size of $\varphi 15 \times 5 \mathrm{~mm}$ by using wire-electrode cutting. Then, those small disks were prepared by polishing one surface of the cylinder samples to mirror-like quality. The wellcontacted diffusion couple was then assembled with the small disks under vacuum at $900^{\circ} \mathrm{C}$ for $90 \mathrm{~min}$ with a load of $10 \mathrm{MPa}$ on a vacuum diffusion bonding and hot pressing system. The diffusion couple was sealed into quartz capsules (evacuated and then back flushed with argon) and then was subjected to long-term diffusion annealing at $1250^{\circ} \mathrm{C}$ for 12 hours followed by quenching in ice water. The diffusion couple was then sectioned in half parallel to the diffusion direction which suffered no oxidation and evaporation of elements and polished by standard metallographic techniques. The local composition was analyzed by electron microprobe analysis (EPMA) on JEOL JXA 8900 at the assigned area.

In order to avoid oxidation, the samples were sealed into quartz capsules again before the secondary heat treatment for introducing the $\beta$ to $\alpha$ phase transformation process. The diffusion couple was first solution treated above $\alpha / \beta$ phase transformation temperature to obtain a fully $\beta$ single-phase microstructure, and then cooling was performed by furnace cooling to $700^{\circ} \mathrm{C}$ followed by water quenching for precipitating the appropriate $\alpha$ phase. Considering the $\alpha / \beta$ phase transformation temperature of each alloy in the Ti5.04Al/Ti-1.52Mo diffusion couple, the solid-solution treatment was performed at $980^{\circ} \mathrm{C}$ for $15 \mathrm{~min}$.

The surface of the Ti-5.04Al/Ti-1.52Mo diffusion couple was then carefully prepared by polishing with several diamond paste of decreasing size followed by a final polishing with a colloidal silica suspension of $0.02 \mu \mathrm{m}$. The precipitation of $\alpha$ in the central diffusion zone was determined by the electron backscatter diffraction (EBSD) using a FEI Helios NanoLab 600i with a HKL-Channel 5 system.

\section{Results and Discussion}

After long-term diffusion annealing at $1250^{\circ} \mathrm{C}$ for 12 hours followed by quenching in ice water, the concentration profiles of the Ti-5.04Al/Ti-1.52Mo diffusion couple are first measured by EPMA, as shown in Figure 1. It can be seen that the long-term diffusion annealing results in a large composition gradient in the diffusion zone in the Ti-5.04Al/Ti$1.52 \mathrm{Mo}$ diffusion couple, which obviously causes the changing of local composition on the $\beta$ matrix. Then, the effect of the changing of local composition of the $\beta$ matrix on the $\alpha$ variant selection can be studied after a secondary heat treatment process based on those composition gradients.

Figure 1 also shows the microstructure in the diffusion zone of the Ti-5.04Al/Ti-1.52Mo diffusion couple after the secondary heat treatment. In the secondary heat treatment process, the solid-solution treatment at $980^{\circ} \mathrm{C}$ results in a fully $\beta$ single-phase microstructure formed in the diffusion zone. Then, the $\alpha$ phase gradually precipitates in the $\beta$ matrix during the furnace cooling process. It can be seen in Figure 1 that the significant microstructure gradients formed in the diffusion zone due to the changing of local composition of the $\beta$ matrix. The volume fraction and width of $\alpha$ gradually decrease from the Ti-5.04Al side to the Ti-1.52Mo side of the diffusion couple.

Because only the orientation information of the $\alpha$ phase in a single $\beta$ grain can be used for the studying the variant selection behavior, a series of small areas are selected for the EBSD test. From those tested EBSD results, the area marked by the red rectangle in Figure 1 is selected for the analysis due to the reason that only one single $\beta$ grain existed in this area, as shown in Figure 2. From the left to right in the red rectangle, the measured composition of $\mathrm{Al}$ decreases from $0.4 \%$ to $0.29 \%$ with an average composition of $0.34 \%$, while the composition of Mo increases from $1.23 \%$ to $1.27 \%$ with an average composition of $1.24 \%$. The pole figure of the $\beta$ matrix in Figure 2(b) confirmed that there is only a single $\beta$ grain. Figure 3 shows the analysis of the pole figures of the $\alpha$ variants, in which it can be seen that all $12 \alpha$ variants were observed in one $\beta$ grain, which means that there is no variant select phenomenon. The $12 \alpha$ variants are confirmed by calculating the orientation relationship of the $\beta$ grain and $\alpha$ variants based on the pole figures in Figures 2(b) and 3 


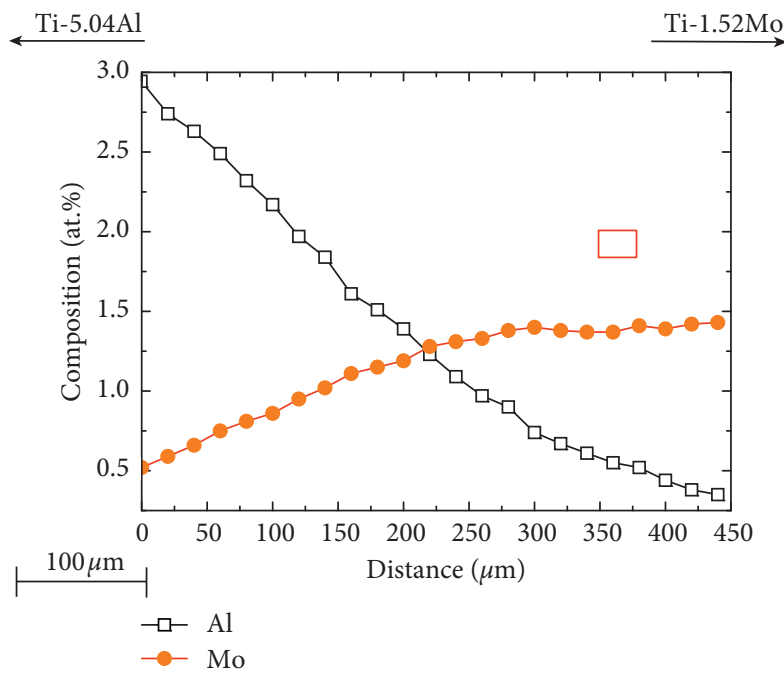

Figure 1: Compositional dependence of the microstructure in the Ti-5.04Al/Ti-1.52Mo diffusion couple after $12 \mathrm{~h}$ annealing at $1250^{\circ} \mathrm{C}$ followed by the secondary heat treatment.

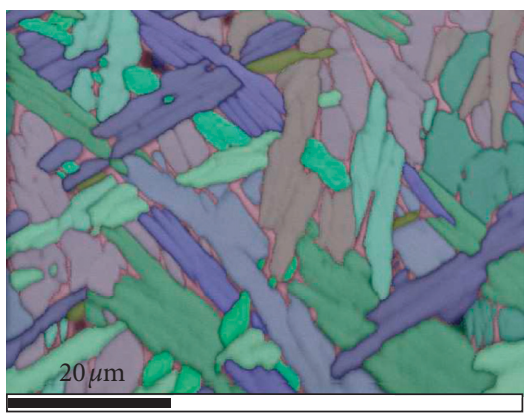

(a)
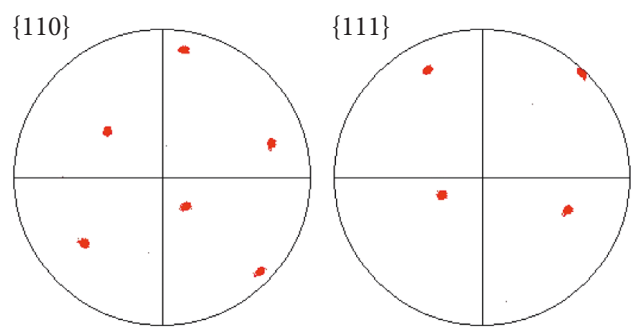

(b)

FIGURE 2: EBSD analysis of the $\alpha$ precipitates in the Ti-5.04Al/Ti-1.52Mo diffusion couple: (a) Euler orientation map; (b) pole figure of the $\beta$ matrix.

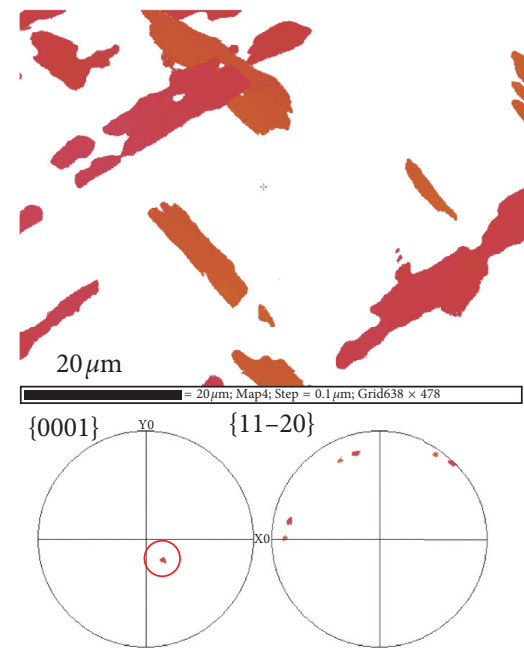

(a)

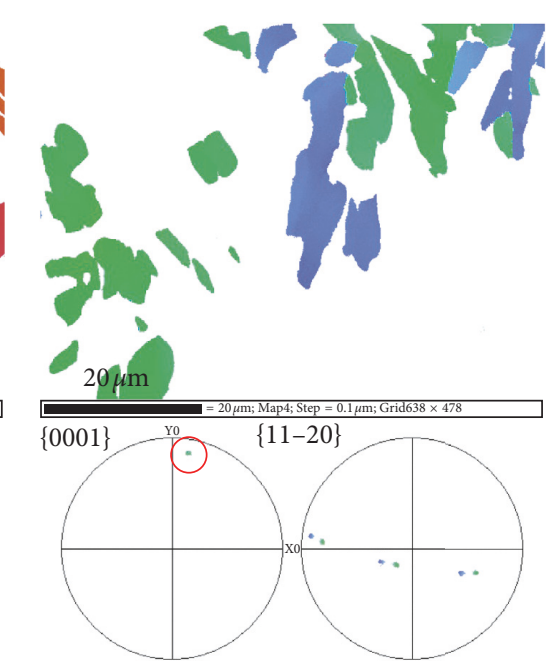

(b)

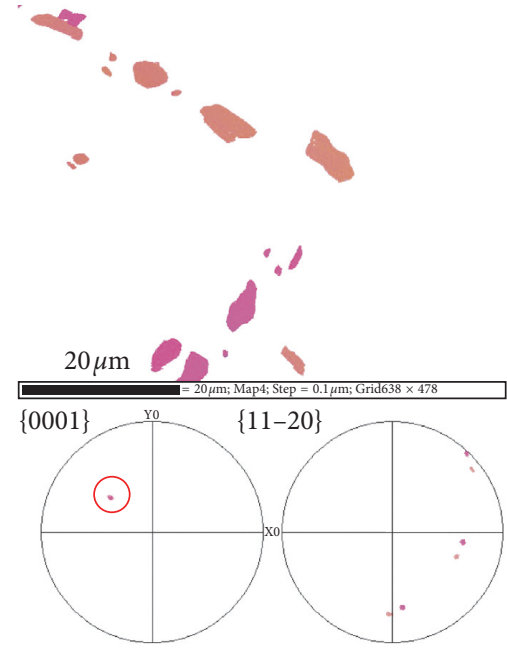

(c)

Figure 3: Continued. 


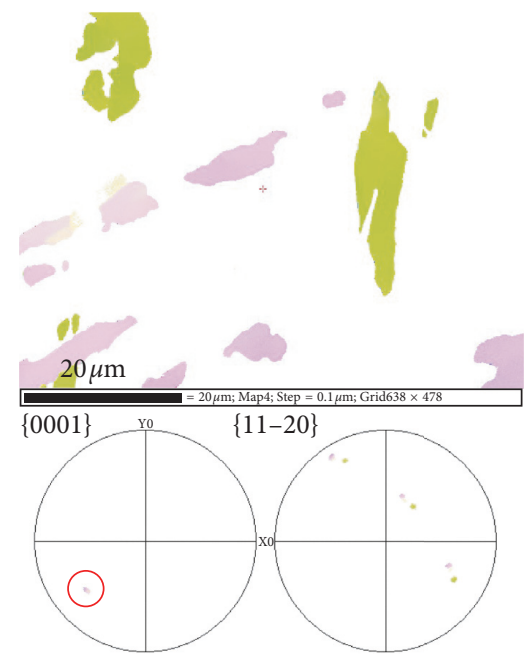

(d)

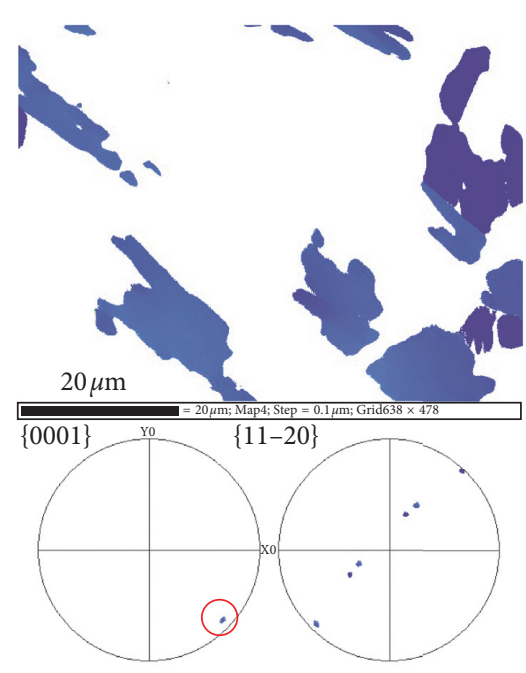

(e)

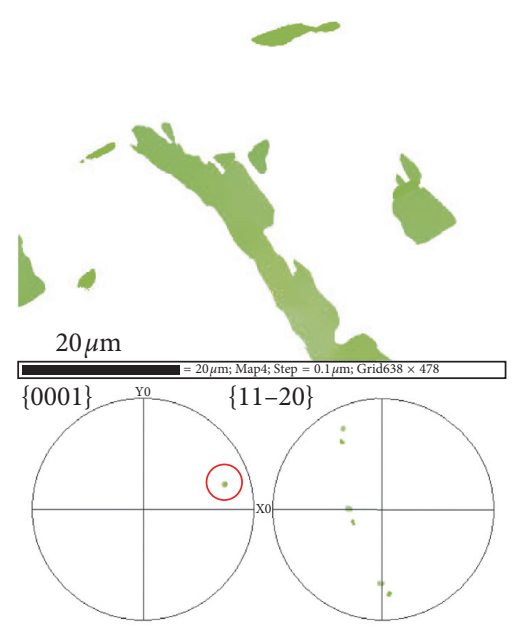

(f)

Figure 3: EBSD analysis of the $12 \alpha$ variants with different orientations in Figure 2.

(Table 1). Through the literature research $[3-5,9,16,17]$, this is the first observation that all 12 variants formed in one $\beta$ grain in titanium alloys during the annealing process.

As in the earlier study [18], the scientists thought that all $\alpha$ variants in a $\beta$ grain occur with equal statistical probability in titanium alloys. However, it is unable to observe all 12 variants in a $\beta$ grain in real alloys, which means the variant selection always takes place during transformation in titanium alloys [9, 18, 19]. The previous work from Shi and Wang [18] and Qui et al [20] conformed that difference of the elastic interaction energy of each variants is the key factor in the formation of the variant selection during $\alpha$ precipitation.

Based on the phase transformation theory [21], the total free energy change during the $\beta$ to $\alpha$ phase transformation process can be expressed by

$$
\Delta G=-\left(V \Delta G_{\mathrm{V}}+\Delta G_{\mathrm{d}}\right)+A \gamma+V \Delta G_{\mathrm{s}},
$$

where $V \Delta G_{\mathrm{V}}$ is the volume free energy reduction, $\Delta G_{\mathrm{d}}$ is the released free energy when nucleation is in the defects, $A \gamma$ is the interfacial energy when the creation of an area $A$ of interface is during the $\beta$ to $\alpha$ phase transformation process, while $V \Delta G_{\mathrm{s}}$ is the elastic energy caused by misfit between the interface of $\beta$ and $\alpha$. From Equation (1), the $\beta$ to $\alpha$ phase transformation can only proceed when the driving force $\left(V \Delta G_{\mathrm{V}}+\Delta G_{\mathrm{d}}\right)$ is large than the activation energy barrier $\left(A \gamma+V \Delta G_{\mathrm{s}}\right)$. When $\alpha$ variants precipitate in the $\beta$ matrix, the elastic energy will be changed due to the different orientation of the $\alpha$ variants $[18,20]$, which will then cause the difference in the activation energy barrier of the different $\alpha$ variants. In titanium alloys, the activation energy barrier of certain $\alpha$ variants is larger than the driving force during the $\beta$ to $\alpha$ phase transformation process, which finally causes the variant selection $[9,18,19]$.

However, this work shows that the $\alpha$ variant selection was eliminated when there has a composition changing in the single $\beta$ grain. The reason can be indicated as follows. In this study, the formation of all $12 \alpha$ variants in one single $\beta$ grain in Figure 3 means that an extra free energy existed in the $\beta$ to $\alpha$ phase transformation in this single $\beta$ grain, which results in the total driving force larger than the activation energy barrier of the all $12 \alpha$ variants. As shown in Figure 1, the composition of the single $\beta$ grain in Figure 2 is gradient changing from one side to another side. The inhomogeneous composition apparently will result in the high free energy in this single $\beta$ grain when comparing with the grains with homogeneous composition, which will cause the increasing of the volume free energy reduction $V \Delta G_{\mathrm{V}}$ in Equation (1), and the driving force will then be increased. The change in gradient composition in this single $\beta$ grain also will accelerate the diffusion of the alloying elements during the $\beta$ to $\alpha$ phase transformation process, which leads to fluctuation in the composition condition of $\alpha$ nucleus more easily, and then, the nucleation of $\alpha$ from $\beta$ matrix can be promoted. Therefore, when the $\alpha$ phase nucleates in this single $\beta$ grain, the extra released free energy caused by the inhomogeneous composition, combined with the accelerated diffusion of the alloying elements, will lead to the total nucleation energy larger than the activation energy barrier of all the $12 \alpha$ variants, after which $12 \alpha$ variants formed.

As a final point, it is very interesting to observe that all 12 $\alpha$ variants simultaneously precipitated in one $\beta$ grain when the composition changed in the $\beta$ matrix, meaning the weakening of variant selection during the $\beta$ to $\alpha$ phase transformation process, which is implicated in the enhance of the fatigue resistance and creep performance of titanium alloys $[13,14]$.

\section{Conclusion}

In this paper, the effect of the changing of the local composition of the $\beta$ matrix on the precipitation of $\alpha$ phase has been investigated by using the Ti-5.04Al/Ti-1.52Mo (at.\%) diffusion couple. 
TABLE 1: Euler angles and Burgers orientation relationship of all 12 variants.

\begin{tabular}{|c|c|c|c|}
\hline Variants & Euler angle & Orientation relationship & 3D orientations \\
\hline BCC & $(151.2,33.8,26.6)$ & & \\
\hline V1 & $(38,21.1,49.3)$ & $(101)_{\beta} / /(0001)_{\alpha}[11 \overline{1}]_{\beta} / /[11 \overline{2} 0]_{\alpha}$ & \\
\hline $\mathrm{V} 2$ & $(37.4,19.6,39.3)$ & $(101)_{\beta} / /(0001)_{\alpha}[\overline{1} 11]_{\beta} / /[11 \overline{2} 0]_{\alpha}$ & \\
\hline V3 & $(171.4,76.4,39.3)$ & $(\overline{1} 01)_{\beta} / /(0001)_{\alpha}[1 \overline{1} 1]_{\beta} / /[11 \overline{2} 0]_{\alpha}$ & \\
\hline V4 & $(170.9,76.1,51)$ & $(\overline{101})_{\beta} / /(0001)_{\alpha}[111]_{\beta} / /[11 \overline{2} 0]_{\alpha}$ & \\
\hline V5 & $(51.1,140.2,36)$ & $(0 \overline{1} 1)_{\beta} / /(0001)_{\alpha}[\overline{1} 11]_{\beta} / /[11 \overline{2} 0]_{\alpha}$ & \\
\hline V6 & $(49.8,140.4,44.8)$ & $(0 \overline{1} 1)_{\beta} / /(0001)_{\alpha}[111]_{\beta} / /[11 \overline{2} 0]_{\alpha}$ & \\
\hline V7 & $(130.7,121.6,43.2)$ & $(1 \overline{1} 0)_{\beta} / /(0001)_{\alpha}[111]_{\beta} / /[11 \overline{2} 0]_{\alpha}$ & \\
\hline V8 & $(130.3,121.8,54.2)$ & $(1 \overline{1} 0)_{\beta} / /(0001)_{\alpha}[11 \overline{1}]_{\beta} / /[11 \overline{2} 0]_{\alpha}$ & \\
\hline V9 & $(47.1,79.5,33.5)$ & $(110)_{\beta} / /(0001)_{\alpha}[\overline{1} 11]_{\beta} / /[11 \overline{2} 0]_{\alpha}$ & \\
\hline V10 & $(46.0,79.2,22.8)$ & $(110)_{\beta} / /(0001)_{\alpha}[1 \overline{1} 1]_{\beta} / /[11 \overline{2} 0]_{\alpha}$ & \\
\hline V11 & $(107.5,65.3,2.7)$ & $(011)_{\beta} / /(0001)_{\alpha}[1 \overline{1} 1]_{\beta} / /[11 \overline{2} 0]_{\alpha}$ & \\
\hline V12 & $(107.5,66.5,51.6)$ & $(011)_{\beta} / /(0001)_{\alpha}[11 \overline{1}]_{\beta} / /[11 \overline{2} 0]_{\alpha}$ & \\
\hline
\end{tabular}


(1) After long-term diffusion annealing, a large composition gradient formed in the diffusion zone in the Ti-5.04Al/Ti-1.52Mo diffusion couple causes the changing of local composition on the $\beta$ matrix. After the secondary heat treatment process, the precipitation of $\alpha$ also shows the gradient variation in the diffusion zone due to the changing of local composition of the $\beta$ matrix.

(2) The first observation that all 12 variants formed in one $\beta$ grain is confirmed by the EBSD analyzing in the diffusion zone in the Ti-5.04Al/Ti-1.52Mo diffusion couple due to the changing of the local composition of the $\beta$ matrix. When the $\alpha$ phase nucleates in this single $\beta$ grain, the extra released free energy caused by the inhomogeneous composition will lead to the total driving force larger than the activation energy barrier of all the $12 \alpha$ variants, combined with the accelerated diffusion of the alloying elements, which finally results in the formation of all $12 \alpha$ variants.

\section{Data Availability}

The data used to support the findings of this study are included within the article.

\section{Conflicts of Interest}

The authors declare that they have no conflicts of interest.

\section{Acknowledgments}

The authors would like to thank the National Natural Science Foundation of China (Grant no. 51601077) and the Natural Science Foundation of Jiangsu Province (Grant no. BK20160291) for the financial support. This work was also supported by the fund of the State Key Laboratory of Solidification Processing in NWPU (Grant no. SKLSP201743) and the Jiangsu Planned Projects for Postdoctoral Research Funds (Grant no. 1701006B).

\section{References}

[1] S. L. Nyakana, J. C. Fanning, and R. R. Boyer, "Quick reference guide for $\beta$ titanium alloys in the 00s," Journal of Materials Engineering and Performance, vol. 14, no. 6, pp. 799-811, 2005.

[2] O. M. Ivasishin, P. E. Markovsky, Y. V. Matviychuk, S. L. Semiatin, C. H. Ward, and S. Fox, "A comparative study of the mechanical properties of high-strength $\beta$-titanium alloys," Journal of Alloys and Compounds, vol. 457, no. 1-2, pp. 296-309, 2008.

[3] G. C. Obasi, J. Q. D. Fonseca, D. Rugg, and M. Preuss, "The effect of $\beta$ grain coarsening on variant selection and texture evolution in a near- $\beta$ Ti alloy," Materials Science and Engineering: $A$, vol. 576, pp. 272-279, 2013.

[4] E. Lee, R. Banerjee, S. Kar, D. Bhattacharyya, and H. Fraser, "Selection of $\alpha$ variants during microstructural evolution in $\alpha / \beta$ titanium alloys," Philosophical Magazine, vol. 87, no. 24, pp. 3615-3627, 2007.
[5] K. Hua, J. Li, H. Kou, J. Fan, M. Sun, and B. Tang, "Phase precipitation behavior during isothermal deformation in $\beta$-quenched near beta titanium alloy Ti-7333," Journal of Alloys and Compounds, vol. 671, pp. 381-388, 2016.

[6] X. Jiang, B. P. Wynne, and J. Martin, "Variant selection in stationary shoulder friction stir welded Ti-6Al-4V alloy," Journal of Materials Science \& Technology, vol. 34, no. 1, pp. 198-208, 2018.

[7] W. G. Burgers, "On the process of transition of the cubicbody-centered modification into the hexagonal-close-packed modification of zirconium," Physica, vol. 1, no. 7-12, pp. 561-586, 1934.

[8] T. Karthikeyan, S. Saroja, and M. Vijayalakshmi, "Evaluation of misorientation angle-axis set between variants during transformation of bcc to hcp phase obeying burgers orientation relation," Scripta Materialia, vol. 55, no. 9, pp. 771-774, 2006.

[9] M. Salib, J. Teixeira, L. Germain, E. Lamielle, N. Gey, and E. Aeby-Gautier, "Influence of transformation temperature on microtexture formation associated with a precipitation at $\beta$ grain boundaries in a $\beta$ metastable titanium alloy," Acta Materialia, vol. 61, no. 10, pp. 3758-3768, 2013.

[10] N. P. Gurao, A. Ali, and S. Suwas, "Study of texture evolution in metastable $\beta$-Ti alloy as a function of strain path and its effect on $\alpha$ transformation texture," Materials Science and Engineering: A, vol. 504, no. 1-2, pp. 24-35, 2009.

[11] T. Karthikeyan, A. Dasgupta, R. Khatirkar, S. Saroja, I. Samajdar, and M. Vijayalakshmi, "Effect of cooling rate on transformation texture and variant selection during $\beta \rightarrow \alpha$ transformation in Ti-5Ta-1.8Nb alloy," Materials Science and Engineering: A, vol. 528, no. 2, pp. 549-558, 2010.

[12] S. L. Semiatin, K. T. Kinsel, A. L. Pilchak, and G. A. Sargent, "Effect of process variables on transformation-texture development in Ti-6Al-4V sheet following beta heat treatment," Metallurgical and Materials Transactions A, vol. 44, no. 8, pp. 3852-3865, 2013.

[13] G. Lütjering and J. C. Williams, Titanium, Springer, Berlin, Germany, 2007.

[14] D. Banerjee and J. C. Williams, "Perspectives on titanium science and technology," Acta Materialia, vol. 61, no. 3, pp. 844-879, 2013.

[15] P. Bate and B. Hutchinson, "The effect of elastic interactions between displacive transformations on textures in steels," Acta Materialia, vol. 48, no. 12, pp. 3183-3192, 2000.

[16] D. Bhattacharyya, G. B. Viswanathan, and H. L. Fraser, "Crystallographic and morphological relationships between $\beta$ phase and the Widmanstätten and allotriomorphic $\alpha$ phase at special $\beta$ grain boundaries in an $\alpha / \beta$ titanium alloy," Acta Materialia, vol. 55, no. 20, pp. 6765-6778, 2007.

[17] D. Bhattacharyya, G. B. Viswanathan, R. Denkenberger, D. Furrer, and H. L. Fraser, "The role of crystallographic and geometrical relationships between $\alpha$ and $\beta$ phases in an $\alpha / \beta$ titanium alloy," Acta Materialia, vol. 51, no. 16, pp. 46794691, 2003.

[18] R. Shi and Y. Wang, "Variant selection during $\alpha$ precipitation in Ti-6Al-4V under the influence of local stress-a simulation study," Acta Materialia, vol. 61, no. 16, pp. 6006-6024, 2013.

[19] R. Shi, V. Dixit, H. L. Fraser, and Y. Wang, "Variant selection of grain boundary a by special prior $\beta$ grain boundaries in titanium alloys," Acta Materialia, vol. 75, pp. 156-166, 2014.

[20] D. Qiu, R. Shi, D. Zhang, W. Lu, and Y. Wang, "Variant selection by dislocations during $\alpha$ precipitation in $\alpha / \beta$ titanium alloys," Acta Materialia, vol. 88, pp. 218-231, 2015. 
[21] D. A. Porter and K. E. Easterling, Phase Transformations in Metals and Alloys, Chapman \& Hall, London, UK, 2nd edition, 1992.

[22] Y. Chen, H. Kou, L. Cheng, Y. Zhang, Y. Yu, and Y. Lu, "Kinetic diffusion couple for mapping microstructural and mechanical data on Ti-Al-Mo titanium alloys," Materials, vol. 11, no. 7, p. 1112, 2018. 


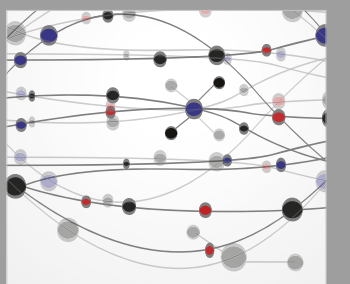

The Scientific World Journal
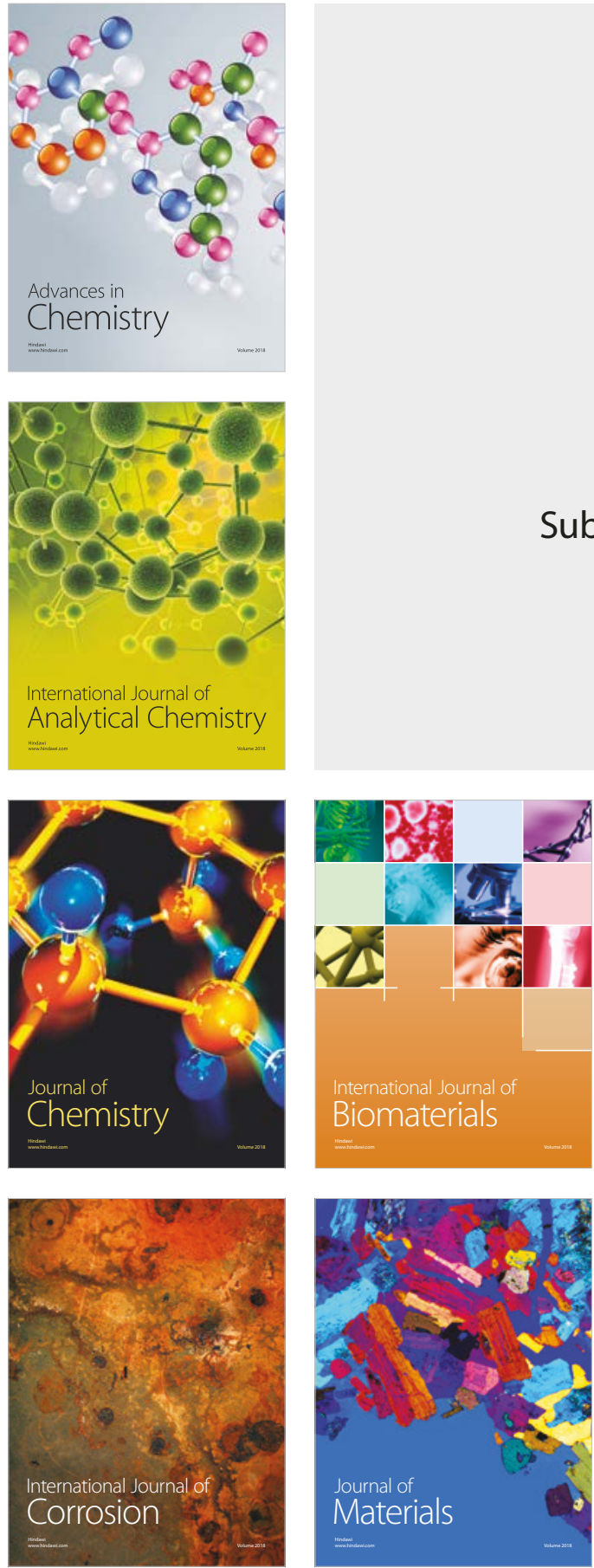

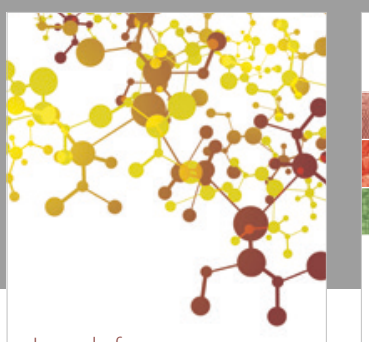

Journal of

Applied Chemistry
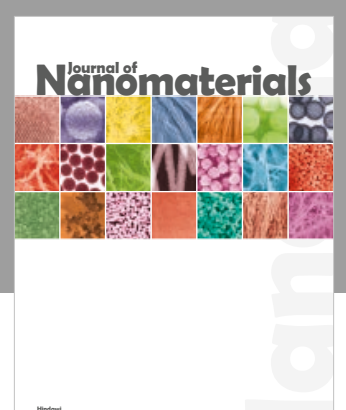

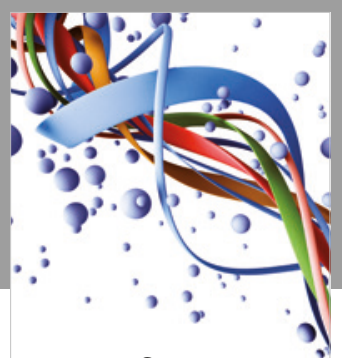

Scientifica

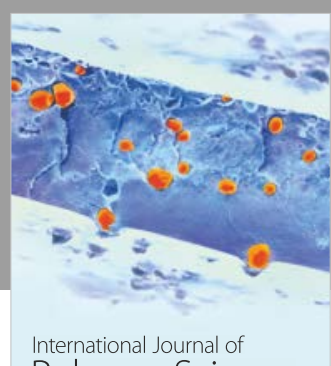

Polymer Science

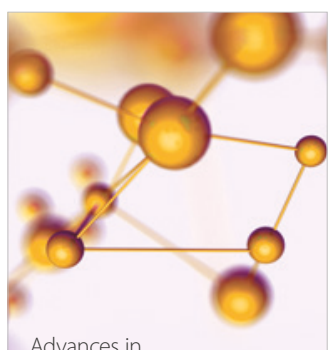

Physical Chemistry
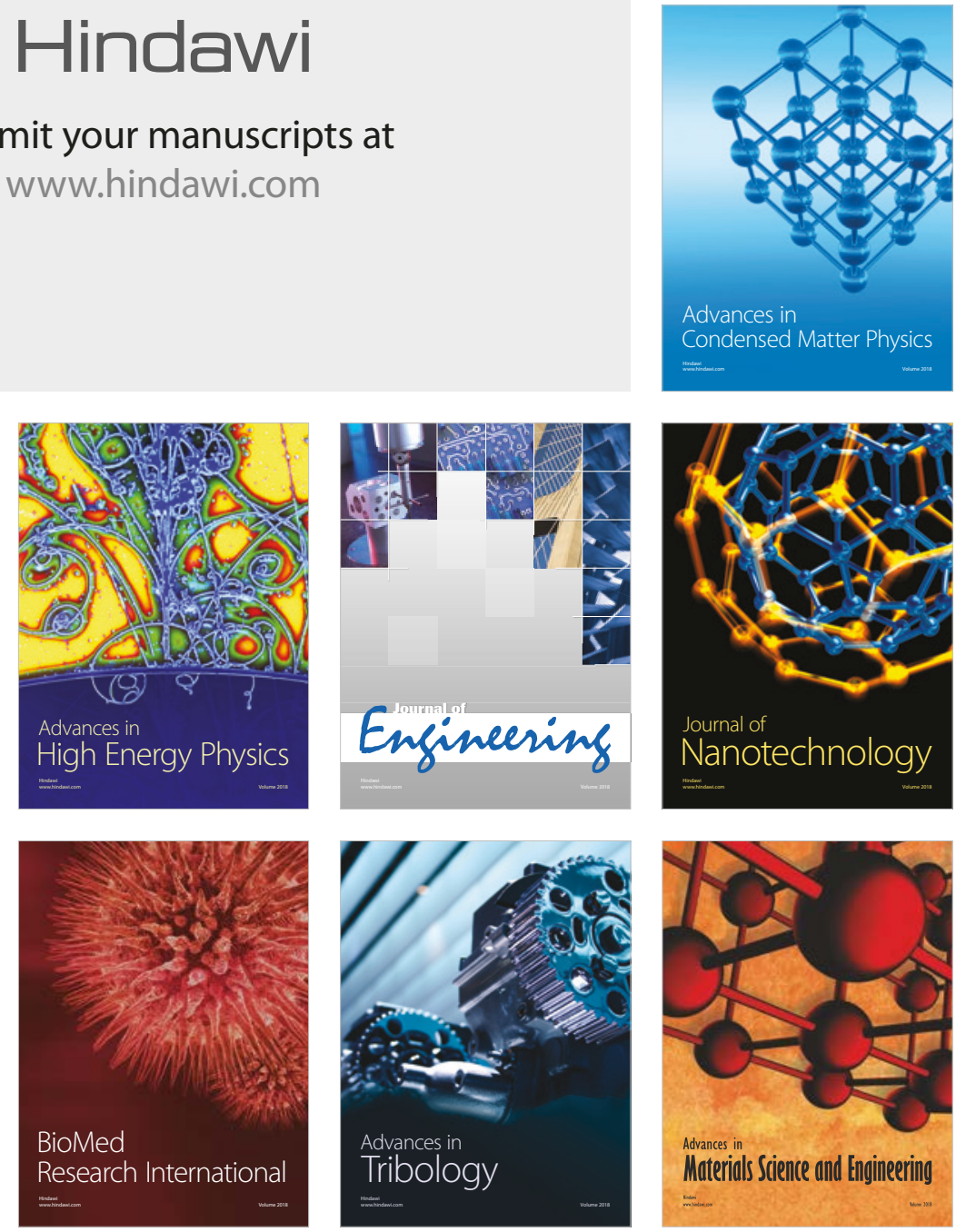